\title{
Functionalized Multiwalled Carbon Nanotubes for UV Coating
}

\author{
Ö.F. Vurur ${ }^{1}$ İ.E. Serhatli2,** \\ ${ }^{1}$ Istanbul Technical University, Institute of Science and Technology, Polymer Science and Technology Programme, \\ Maslak, 34469, Istanbul, Turkey \\ ${ }^{2}$ Istanbul Technical University, Faculty of Science and Letters, Chemistry Department, Maslak, 34469, Istanbul, \\ Turkey
}

(Received 18 July 2013; published online 29 August 2013)

\begin{abstract}
We propose a strategy to enhance the photocurable properties of the multi walled carbon nanotube (Mwcnt) / binder films through control of the intermolecular interaction between the nanotube and the binder material. For this aim, photosensitive functionalized carbon nanotube was synthesized and by mixing binder, it was cured under UV-light. Epoxy acrylate resin was used as binder and reactive diluents such as HDDA, DPGDA were added as both crosslinker and viscosity extender.
\end{abstract}

Keywords: Carbon nanotubes, Functionalized multiwalled carbon nanotubes, UV Coating.

PACS numbers: 81.07.De, 82.35.Np

\section{INTRODUCTION}

Carbon nanotubes have generated tremendous scientific and technical interest over the past decade due to their unique mechanical and physical properties at the nanoscale [1]. Chemical modification and covalent functionalization of CNTs with several organic species like alcohols, amines and polymers can be done in many ways as reported elsewhere [2,3]. The generation and functionalization of defect sites at the end or side walls of the nanotubes by oxidation is a well known exohedral functionalization technique. The defect sites on the surface of the carbon nanotubes, which upon the treatment under oxidative conditions are transferred to carboxylic acid moieties, allow covalent linkages of oligomers or polymers with the nanotubes $[4,5]$. It is well known that $\mathrm{HNO}_{3} / \mathrm{H}_{2} \mathrm{SO}_{4}$ mixture, as an oxidizing agent, creates an open end termini in the structure that are stabilized by $-\mathrm{COOH}$ and $\mathrm{OH}$ groups left bonded to the nanotubes at the end termini and/or the sidewall defect sites [5-6]. The combination of their mechanical and physical properties makes carbon nanotubes potentially ideal for use as sensing materials [7, 8]. Most of the photosensitive CNT pastes developed to date are composed of CNTs along with an organic binder such as ethyl cellulose or acrylic polymer, and a photoinitiator. The organic vehicle in photosensitive paste is photopolymerized by UV exposure to produce a negative pattern through the developing process [9-11]. In this study, photosensitive functionalized carbon nanotube was synthesized. By mixing with epoxyacrylate it was cured under UV-light. The characterizations of obtained films were examined.

\section{EXPERIMENTAL PART}

According to procedure for chemical oxidation of carbon nanotubes, $4 \mathrm{gr}$ pure Mwnct was added into $250 \mathrm{ml}$ round-bottom flask equipped with a condenser, $\mathrm{H}_{2} \mathrm{SO}_{4}$ and $\mathrm{HNO}_{3}$ (3:1 by volume ) were poured into flask slowly and mixture was sonicated in ultrasonic bath for $10 \mathrm{~min}$. Then flask was heated to $100{ }^{\circ} \mathrm{C}$ and reaction was performed for $100 \mathrm{~min}$. After cooling down to room temperatire, the product was diluted with water and filtered by using 0,45 micron PTFE membran filter via filtration apparatus. The dilution process was repeated until $\mathrm{pH}$ value reached at 7 . And product was dried in vacuum oven. The oxidized carbon nanotubes were acylated with thionyl chloride. The acylation reaction was applied under reflux at $75{ }^{\circ} \mathrm{C}$ for $48 \mathrm{~h}$. After reaction was completed, the product was filtered with PTFE membrane filter and washed with THF to remove unreacted thionyl chloride. Acylated CNTs were dried in vacuum oven. For synthesis of photosensitive groups attached to CNTs, acyl chloride group containing CNTs were reacted with 2-hydroxy ethyl methacrylate (HEMA). The reaction was performed at $65^{\circ} \mathrm{C}$ for $24 \mathrm{~h}$ by using pyridine as catalyst. Then product was filtered, washed wtih THF to remove unreacted HEMA and dried in vacuumoven.

\section{RESULT AND DISCUSSION}

Film formulations were perfomed according to Table 1:

Epoxy acrylate: It was used as binder

DPGDA: Dipropylene glycol diacrylate. It was used as both reactive diluent and crosslinker agent.

HDDA: Hexanediol diacrylate. It was used as both reacrive diluent and crosslinker agent.

Irgacure 184: Photoinitiator.

HEMA-Mwcnt: Monomer was added to formulation in the range of 0,1 to 1 .

In order to evaulate the coating performance, each formulation was applied on teflon mould panels and cured in a UV Processor ( EMA, $120 \mathrm{~W} / \mathrm{cm}^{2}$, medium pressure mercury lamps).

FT-IR characterizations of functionalized carbon nanotubes were performed with dispersion method. Because carbon nanotubes are black, they have the property of absorbing laser light coming from FT-IR

\footnotetext{
*serhatli@itu.edu.tr
} 
source. Therefore, IR charaterization has been difficult. In dispersion method, carbon nanotubes have dispersed in THF so peaks of functional group have been obtained.

Table 1 - Acrylated carbon nanotube containing film formulations (AFC) as weightly

\begin{tabular}{|c|c|c|c|c|c|}
\hline $\mathrm{w} / \mathrm{w}$ & $\begin{array}{c}\text { Epoxy } \\
\text { Acrylate }\end{array}$ & dpgda & hdda & $\begin{array}{c}\text { Irga- } \\
184\end{array}$ & $\begin{array}{c}\text { MWCNT- } \\
\text { hema }\end{array}$ \\
\hline $\begin{array}{c}\mathrm{ACF} \\
0\end{array}$ & 60 & 25 & 12 & 3 & - \\
\hline $\begin{array}{c}\mathrm{ACF} \\
1\end{array}$ & 60 & 24,9 & 12 & 3 & 0,1 \\
\hline $\begin{array}{c}\mathrm{ACF} \\
2\end{array}$ & 60 & 24,5 & 12 & 3 & 0,5 \\
\hline $\begin{array}{c}\mathrm{ACF} \\
3\end{array}$ & 60 & 24 & 12 & 3 & 1 \\
\hline
\end{tabular}

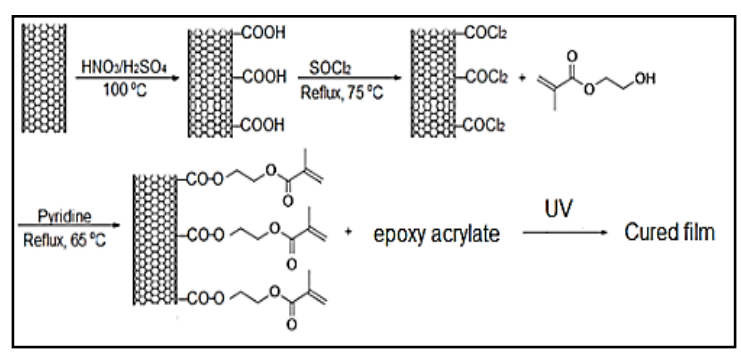

Fig.1- Synthesis of nanocoating

FT-IR characterizations of functionalized carbon nanotubes were performed with dispersion method. Because carbon nanotubes are black, they have the property of absorbing laser light coming from FT-IR source. Therefore, IR charaterization has been difficult. In dispersion method, carbon nanotubes have dispersed in THF so peaks of functional group have been obtained. According to IR spectrum of THF, aliphatic $\mathrm{CH}_{2}$ groups are observed at about $2800-2900 \mathrm{~cm}^{-1}$. Etheric bond $\mathrm{C}-\mathrm{O}$ appears at $1066 \mathrm{~cm}^{-1}$. For other spectrums, THF is accepted as baseline peak.

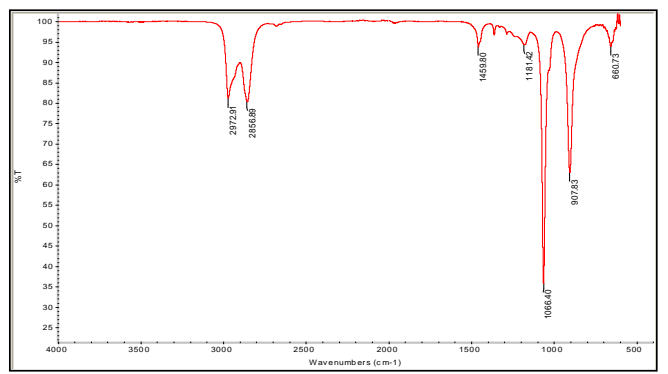

Fig. 2 - FT-IR spectrum of THF

For Fig. 4, peak at $1737 \mathrm{~cm}^{-1}$ corresponds to $\mathrm{C}=\mathrm{O}$ carbonyl bond, broad peak at about $3500 \mathrm{~cm}^{-1}$ belongs to $-\mathrm{OH}$ group of oxidized carbon nano tubes. It is clearly seen that carboxyl groups are attached onto carbon nanotube surface by covalent bond.

According to thermograms, pure carbon nanotube is thermally stable up to $550^{\circ} \mathrm{C}$. The weight loss of oxidized carbon nanotube at $550{ }^{\circ} \mathrm{C}$ is about $10,1 \%$ and the weight loss of acrylated carbon nanotube at $550{ }^{\circ} \mathrm{C}$ is about $15 \%$.

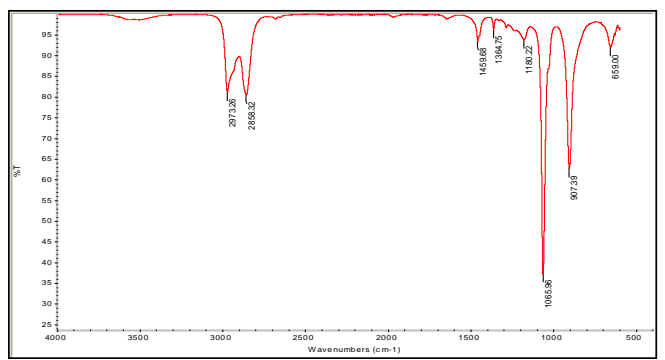

Fig. 3 - FT-IR spectrum of pure carbon nanotube

IR spectrum of pure carbon nanotubes is same as those of THF. Small peak at about $3500 \mathrm{~cm}^{-1}$ which is negligible can be moisture.

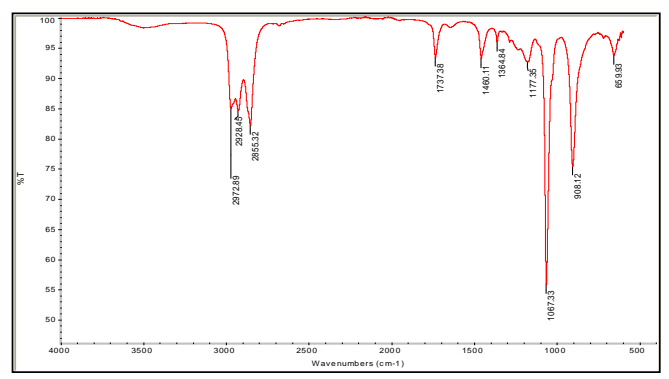

Fig. 4 - FT-IR spectrum of carboxylated carbon nanotube

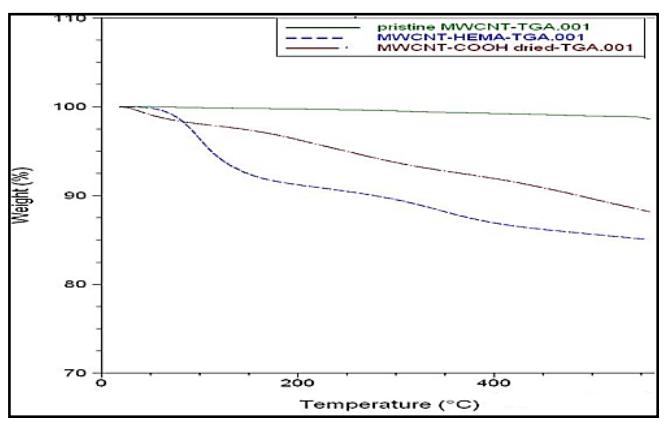

Fig. 5 - TGA thermograms of carbon nanotubes

The morphology and structure of pure-Mwcnt and carboxylated-Mwcnt were investigated by SEM (Fig. 6 and 7). Pure Mwcnt present a smooth surface and a loosely packed arrangement. The image of carboxylated-Mwcnt shows not only that the length of nanotubes wss reduced by the strong acid treatment but also that the carboxylated carbon nanotube are highly tangled with each other.

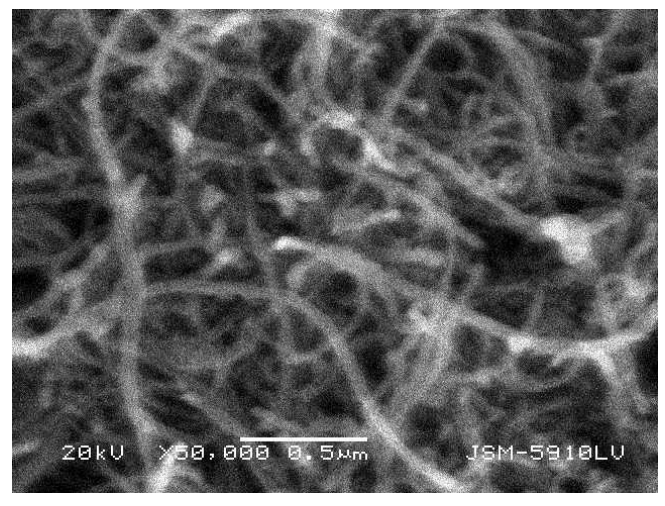

Fig. 6 - SEM image of pure CNT 


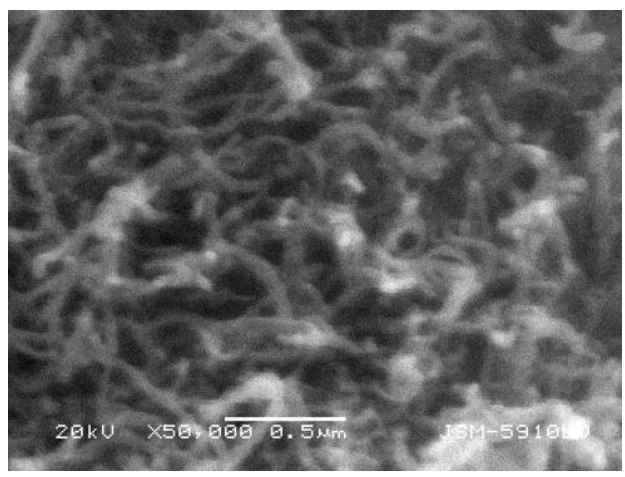

Fig. 7 - SEM image of carboxylated CNT

For cured film, mechanical and gel content tests were performed. As seen from tensile test results, ACF1 shows the best results compared to other ones, and it can be said that adding the acrylated-CNT to curable epoxyacrylate formulations leads to increase excellent modulus and tensile strength. Also one reaches a conclusion that optimum value to add acrylated-CNT for formulation is $0,1 \%$.

Table 2 - Tensile test for acrylated carbon nanotube films

\begin{tabular}{|c|c|c|c|}
\hline $\begin{array}{c}\text { Sample } \\
\text { Name }\end{array}$ & $\begin{array}{c}\text { Modulus } \\
\text { (Mpa) }\end{array}$ & $\begin{array}{c}\text { Tensile Test at } \\
\text { break (Mpa) }\end{array}$ & $\begin{array}{c}\text { Tensile Strain } \\
\text { at break (\%) }\end{array}$ \\
\hline ACF 0 & 601,02 & 3,2 & 6,89 \\
\hline ACF 1 & 922,6 & 30,18 & 5,34 \\
\hline ACF 2 & 826,73 & 10,42 & 5,25 \\
\hline ACF 3 & 809,02 & 8,35 & 5,6 \\
\hline
\end{tabular}

\section{REFERENCES}

1. E.T. Thostenson, C.Y. Li, T.W. Chou, Compos. Sci. Technol. 65, 491 (2005).

2. P.M. Ajayan, Chem. Rev. 99, 1787 (1999).

3. Y. Lin, M.J. Meziani, Y.P. Sun, J. Mater. Chem. 17, 1143 (2007).

4. J.E. Riggs, Z.X. Guo, D.L. Carroll, Y.P. Sun, J. Am. Chem. Soc. 122, 5879 (2000).

5. Y. Lin, A.M. Rao, B. Sadanadan, E.A. Kenik, Y. Sun, J. Phys. Chem. B 106, 1294 (2002).

6. F.J. Gomez, R.J. Chen, D. Wang, R.M. Waymouth, H. Dai, Chem. Commun. 190, (2003).

7. B. Mahar, C. Laslau, R. Yip, Y. Sun, IEEE Sens. J. 7, 266 (2007).
For gel content measurement, value of ACF1 is the same as ACF0 and it can be concluded that ACF1 has the optimun condition for film formulation.

Table 3 - Gel content results

\begin{tabular}{|c|c|}
\hline Sample Name & Gel Content (\% w ) \\
\hline ACF 0 & 98,1 \\
\hline ACF 1 & 98 \\
\hline ACF 2 & 97 \\
\hline ACF 3 & 96 \\
\hline
\end{tabular}

\section{CONCLUSION}

Multiwalled carbon nanotubes (Mwcnt) were covalently functionalized with - $\mathrm{COOH}$ group and $\mathrm{C}=\mathrm{C}$ double bond containing HEMA And photocurable properties of Mwcnt-HEMA/binder system were examined. The linkage of carboxylated group can be clearly seen from FTIR spectrums and SEM images. According to tensile test and gel content test results for prepared film formulations, it is observed good result for the film containing Mwcnt-HEMA $0,1 \% \mathrm{w} / \mathrm{w}$ compared to film not containing MwcntHEMA. But adding excessive amount of functionalized carbon nanotubes causes decrease in modulus and tensile strength and it can be said that carbon nanotubes are not completely alignment due to their funtionalized structure.

8. C. Li, E.T. Thostenson, T.W. Chou, Compos. Sci. Technol. 68,1227 (2008).

9. J.-H. Park, J.S. Moon, J.W. Nam, J.B. Yoo, C.Y. Park, J.M. Kim, J.H. Park, C.G. Lee, D.H. Choe, Diamond Relat. Mater. 14, 2113 (2005).

10. Y.S. Choi, Y.S. Cho, J.H. Kang, Y.J. Kim, I.H. Kim, S.H. Park, H.W. Lee, S.Y. Hwang, S.J. Lee, C.G. Lee, T.S. Oh, J.S. Choi, S.K. Kang, J.M. Kim, Appl. Phys. Lett. 82, 3565 (2003).

11. J.-H.Park, G.H. Son, J.-S. Moon, J.H. Han, A.S. Bardinsky, D.G. Kuvshinv, J.-B. Yoo, C.-Y. Park, J.W. Nam, J. Park, C.G. Lee, D.H. Choe, J. Vac. Sci. Technol. B 23, 749 (2005). 